\title{
An Effective One-pot Synthesis of 5-Substituted Tetronic Acids.
}

David Tejedor Aragón, ${ }^{\not \#}$ Gloria V. López, ${ }^{\dagger}$ Fernando García-Tellado, ${ }^{\dagger \pm *}$ José Juan MarreroTellado, ${ }^{\text {\#\# Pedro de Armas }}{ }^{\dagger \pm}$ and David Terrero ${ }^{\dagger}$

Instituto de Productos Naturales y Agrobiología, CSIC, Avda. Astrofísico Francisco Sánchez 3, 38206 La Laguna, Tenerife, Spain; Instituto Universitario de Bio-orgánica Antonio González, Universidad de La Laguna, Avda. Astrofísico Francisco Sánchez 2, 38206 La Laguna, Tenerife, Spain; Instituto Canario de Investigación del Cáncer, Avda. Astrofísico Francisco Sánchez 2, 38206 La Laguna, Tenerife, Spain

dtejedor@ipna.csic.es, $\quad$ vlopez@fq.edu.uy, $\quad$ jdterrero@hotmail.com, $\quad$ fgarcia@ipna.csic.es, jtellado@ull.es, parmas@ipna.csic.es,

RECEIVED DATE (to be automatically inserted after your manuscript is accepted if required according to the journal that you are submitting your paper to)

\begin{abstract}
An expeditious one-pot synthesis of 5-substituted tetronic acids from aldehydes and terminal conjugated alkyne as starting materials is described. The entire process embodies two consecutive chemical events: a catalytic domino reaction to build the 1,3-dioxolane scaffolds 5 and a two-step acidcatalyzed trans-acetalization - lactonization reaction to furnish the tetronic acid derivatives 6.
\end{abstract}

Keywords. Tetronic acids, 5-substituted, one-pot synthesis, 1,3-dioxolanes, domino reactions, acetylides, non-metallated, catalytic, alkynilides. 
Tetronic acids (4-hydroxy-5H-furan-2-one) form a subclass of $\beta$-hydroxybutenolides with the generic structure $1 .{ }^{1}$ The best known members of this family are vitamin C (ascorbic acid) 2 and pennicillic acid 3. A great number of these compounds and their metabolites are found in many natural products, which exhibit a wide array of biological properties. ${ }^{1,2}$

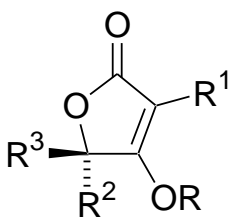

$1 \quad \mathrm{R}=\mathrm{R}^{1}=\mathrm{R}^{2}=\mathrm{R}^{3}=\mathrm{H}$

$2 \mathrm{R}=\mathrm{R}^{2}=\mathrm{H} ; \mathrm{R}^{1}=\mathrm{OH}$;

$\mathrm{R}^{3}=\mathrm{CH}(\mathrm{OH}) \mathrm{CH}_{2} \mathrm{OH}$

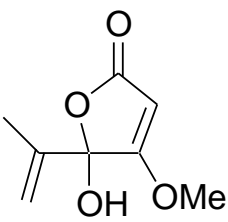

3

Several strategies have been used for the preparation of 5-substituted tetronic acids. Most of them utilize either a Dieckmann reaction ${ }^{2 \mathrm{a}, 3}$ or a cyclization of a suitable $\beta$-ketoester derivative bearing a $\gamma$ halogen atom ${ }^{4}$ or a $\gamma$-oxygenated function. ${ }^{5,6,7}$ Other methods utilize ketenes to generate the $\gamma$-lactonic ring. ${ }^{8} \quad$ Also, 1,3-dioxola-4-ones, ${ }^{9}$ 2-dioxolanones ${ }^{10}$ and substituted 3 -furanones ${ }^{11}$ have been used as templates in the synthesis of these molecules. To the best of our knowledge, a one-pot synthesis of tetronic acid derivatives using cheap, commercially available and non-elaborated starting materials has not been described. ${ }^{12}$ We report here on a simple, general and effective one-pot method to obtain 5substituted tetronic acids. The method comprises two consecutive processes: a catalytic domino reaction to build the 1,3-dioxolane intermediates $\mathbf{5}^{13}$ and a two-step acid-catalyzed trans-acetalizationlactonization reaction to furnish the tetronic acid derivatives 6 (Scheme 1).

Domino processes, ${ }^{14}$ when they are performed in a catalytic manner, constitute a powerful and economical synthetic way to introduce chemical and structural complexity. The search for and development of this kind of chemical processes is a great challenge in organic and medicinal chemistry. ${ }^{15}$ Recently, we described ${ }^{13}$ a domino process based on the catalytic generation of a reactive conjugated acetylide by the Michael addition of triethylamine to the terminal conjugated alkynoate in the presence of 
SCHEME 1. One-pot synthesis of 5-substituted tetronic acids

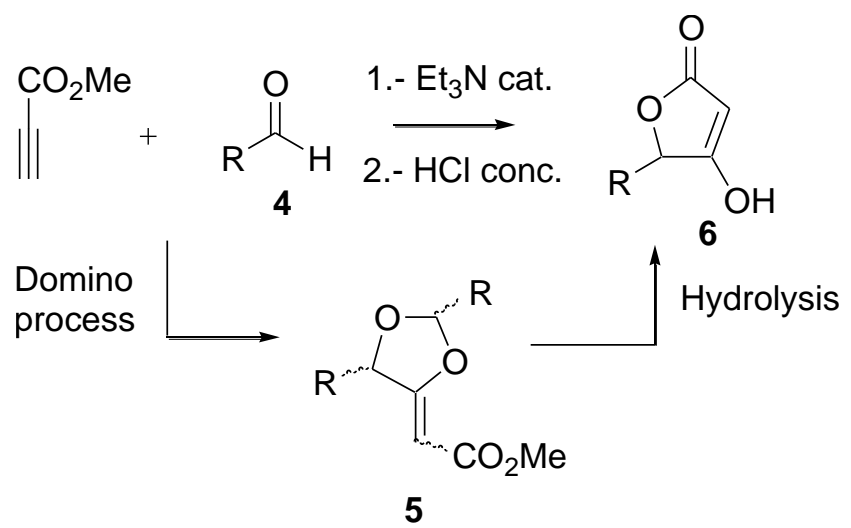

an aldehyde. The key to this system is the low pKa value of these terminal conjugated alkynoates $(\mathrm{pKa}<18.8) .{ }^{16}$ The whole process is outlined in Scheme $2 \mathrm{a}$. The generated ammonium acetylide $\mathbf{I}$ reacts with one molecule of aldehyde to give the ammonium alkoxide II which, in turn, reacts with another molecule of aldehyde to furnish the intermediate vinyl ammonium III. This anion deprotonates to the starting alkynoate generating the 1,3-dioxolane $\mathbf{5}$ and acetylide $\mathbf{I}$ which reinitiates the cycle. This domino process builds up the 1,3-dioxolane intermediates 5 in excellent yields and high efficiency. The synthesis of 5 involves the creation of two $\mathrm{C}-\mathrm{O}$ bonds and one $\mathrm{C}-\mathrm{C}$ bond with both atom-economy and structure-economy. These 1,3-dioxolanes 5 are suitable scaffolds to generate the 3 -oxo- $\gamma$-lactone ring core of the tetronic acids with different patterns of substitution at C-5. Thus, simple trans-acetalization liberates the required $\gamma$-hydroxy $\beta$-ketoester intermediates, which lactonize to furnish the tetronic acid derivatives 6 (Scheme 2b). Tetronic acid derivatives are quite reactive toward aldehydes to give dilactone compounds $7 .{ }^{17}$ Because the trans- acetalization reaction liberates one equivalent of aldehyde, formation of dilactones 7 can involve a serious loss of product. Fortunately, under controlled conditions, 1,3-dioxolanes 5 selectively furnish tetronic acid derivatives $\mathbf{6}$. When both processes are carried out in the same flask, without the isolation of intermediates, in a one-pot fashion, the resulting chemical system constitutes a very appealing reaction manifold for the synthesis of substituted tetronic acid derivatives (See Table 1). The scope and effectiveness of the method is demonstrated by the results 
given in Table 1. 5-Alkyl substituted tetronic acid derivatives are quite accessible regardless off the ramification grade of the alkyl

SCHEME 2. One pot synthesis of the tetronic acid derivatives 6 .

a) Domino process

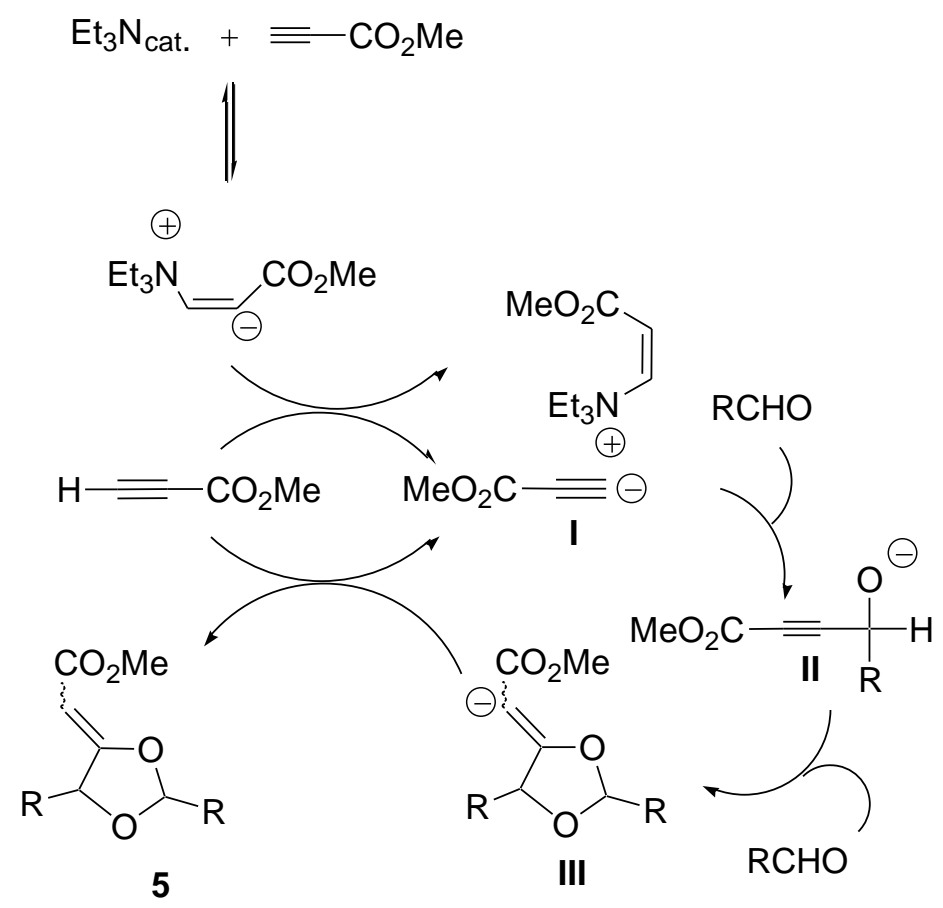

b) Acid-catalyzed trans-acetalization - lactonization<smiles>[R]C(O)/C(=C\C(=O)OC)O[In]C</smiles><smiles>[R]C1OC(=O)C(C([R])C2=C(O)C([R])OC2=O)=C1O</smiles>

7

chain (Entries 1-4). Aldehydes $4 \mathbf{a}$ and $\mathbf{4 b}$ require $24 \mathrm{~h}$ of acid-catalyzed alcoholysis to yield the expected tetronic derivatives $\mathbf{6 a}$ and $\mathbf{6 b}$ in good yields. After $24 \mathrm{~h}$, a remaining amount of dioxolanes $\mathbf{5}$ $(6 \%)$ is still present in the reaction medium and dilactone $7(5 \%)$ begins to appear. Longer reaction 
times lower the tetramate yield favoring the dilactone production. In the case of the more sterically demanding aldehydes $\mathbf{4 c}$ and $\mathbf{4 d}$, an excess of aldehyde (4 equivalents) and longer reaction time for the acid-catalyzed trans-acetalization of the dioxolane intermediates are required to improve the yield from

a

TABLE 1.

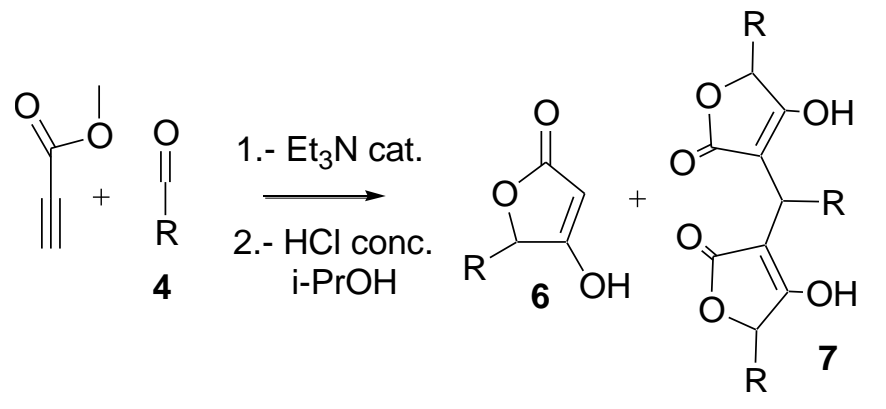

\begin{tabular}{ccccccc}
\hline & & & & \multicolumn{3}{c}{$\mathbf{( \% )}^{\text {a,b }}$} \\
Entry & & $\mathbf{R}$ & & $\mathbf{A}$ & $\mathbf{B}$ & $\mathbf{C}$ \\
\hline 1 & $\mathbf{4 a}$ & $\mathrm{Me}$ & $\mathbf{6 a}^{3 \mathrm{f}}$ & $\mathbf{6 9}$ & & \\
& & & $\mathbf{7 a}$ & $\mathbf{5}$ & & \\
2 & $\mathbf{4 b}$ & $\mathrm{n}-\mathrm{Pr}$ & $\mathbf{6 b}$ & $\mathbf{6 5}$ & & \\
& & & $\mathbf{7 b}$ & $\mathbf{5}$ & & \\
3 & $\mathbf{4 c}$ & i-Pr & $\mathbf{6 c}$ & & 58 & $\mathbf{6 5}$ \\
4 & $\mathbf{4 d}$ & t- $-\mathrm{Bu}$ & $\mathbf{6 d}$ & 47 & 56 & $\mathbf{6 2}$ \\
5 & $\mathbf{4 e}$ & $\mathrm{CH}_{2}=\mathrm{CH}\left(\mathrm{CH}_{2}\right)_{2}$ & $\mathbf{6 e}$ & $\mathbf{5 8}$ & & \\
6 & $\mathbf{4 f}$ & $\mathrm{BnOCH}{ }_{2}$ & $\mathbf{6 f}$ & $\mathbf{4 8}$ & &
\end{tabular}

${ }^{a}$ Yields based on starting alkynoate. ${ }^{b}$ Methods $\mathbf{A}, \mathbf{B}$ and $\mathbf{C}$ are described in the experimental part. ${ }^{\mathrm{c}}$ Starting aldehyde was recovered in the form of its diisopropyl acetal (42\%).

modest $45-50 \%$ to a better $60-65 \%$ (Entries 3,4). In the reaction with isobutanal, the amount of unreacted dioxolane $\mathbf{5 c}$ obtained decreases from $18 \%$ at $24 \mathrm{~h}$ to $10 \%$ at $72 \mathrm{~h}$. Similarly, unreacted $\mathbf{5 d}$ decreases from $13 \%$ to $5 \%$. In both cases, there is no significant formation of the corresponding dilactones due to the steric volume of the $\mathrm{R}$ group in these aldehydes. Tetramates featuring a functionalized alkyl chain in the form of a terminal double bond can be synthesized in good yields from the appropriate aldehyde (Entry 5). This functionality can be further used to elaborate more complex 
molecules or to join this molecule to other biologically active structures. ${ }^{18}$ The biologically relevant 5 hydroxymethyl derivatives ${ }^{19}$ are obtained in good yields with partial recovery of the starting aldehyde in the form of its dialkyl acetal (Entry 6). Aromatic aldehydes are not reactive enough to be used as starting materials in these processes. 2,5-Diaryl substituted dioxolane intermediates $\mathbf{5}$, if formed, do not give a clean and synthetically useful acid-catalyzed trans-acetalization-lactonization reaction.

In summary, we have developed an effective one-pot synthesis of 5-substituted tetronic acid derivatives using methyl propiolate and aldehydes as starting materials. The method embodies two consecutive chemical events: a quite efficient and atom-economy domino process followed by an effective and controlled acid-catalyzed trans-acetalization.

\section{Experimental Section}

Melting points are uncorrected. ${ }^{1} \mathrm{H}$ NMR and ${ }^{13} \mathrm{C}$ NMR spectra of $\mathrm{CDCl}_{3}$ solutions were recorded either at 200 and $50 \mathrm{MHz}$ or at 500 and $125 \mathrm{MHz}$ respectively. FT-IR spectra were measured in chloroform solutions. Flash column chromatography was carried out with silica gel (particle size less than $0.020 \mathrm{~mm}$ ) using appropriate mixtures of ethyl acetate and hexanes as eluent. Dichloromethane was distilled from $\mathrm{CaH}_{2}$. Triethylamine was distilled from potassium hydroxide pellets. All other materials were obtained from commercial suppliers and used as received.

Products 6a-6f exist in solution as an equilibrium of the keto and enol tautomers. The solutions used to obtain the NMR spectra of products $\mathbf{6 a - 6 e}$ contain predominantly the enol form, therefore the spectral data correspond to this tautomer. On the other hand, the solution used to obtain the NMR spectra of product $\mathbf{6} \mathbf{f}$ contains predominantly the other tautomers, therefore the spectral data correspond to the keto form.

\section{General Procedure.}

Method A. Triethylamine $(0.6 \mathrm{mmol})$ was added to a cooled $\left(-78^{\circ} \mathrm{C}\right)$ solution of methyl propiolate $(3$ mmol) and aldehyde $(6.3 \mathrm{mmol})$ in dry $\mathrm{CH}_{2} \mathrm{Cl}_{2}(3 \mathrm{ml})$. The reaction mixture was stirred for $2 \mathrm{~h}$ at this temperature. Concentrated $\mathrm{HCl}\left(0.2 \mathrm{ml}, \approx 2.5 \mathrm{mmol}_{\text {of }} \mathrm{H}^{+}\right)$and isopropanol $(57 \mathrm{ml})$ were added and the 
resulting solution was heated at $60^{\circ} \mathrm{C}$ for $24 \mathrm{~h}$. Evaporation of the solvent at reduced pressure followed by flash chromatography (eluent gradiant: ethyl acetate/hexane from 2:8 to 6:4) yielded tetronic acid derivatives 6a-g as crystalline compounds. The amount of concentrated acid and isopropanol was carefully studied so that the maximum yield of tetronic acids was obtained with respect to the formation of dilactones.

Method B. Same as A but using a larger excess of aldehyde (12 mmol)

Method C. Same as A, but using a longer trans-acetalization time (72 h) under more concentrated conditions ( $25 \mathrm{ml}$ of i-PrOH).

4-Hydroxy-5-methyl-5H-furan-2-one (6a). Mp 113-114 ${ }^{\circ} \mathrm{C}\left(\right.$ Lit. $\left.^{3 \mathrm{f}} 112-115^{\circ} \mathrm{C}\right)$. Dilactone 7a: Mixture of diastereomers. Mp 176.5-180.0 ${ }^{\circ} \mathrm{C} .{ }^{1} \mathrm{H}$ NMR $\left(\mathrm{CDCl}_{3}, 400 \mathrm{MHz}\right): \delta 1.35(\mathrm{~d}, 3 \mathrm{H}, J=7.3 \mathrm{~Hz}), 1.50(\mathrm{~d}$, $6 \mathrm{H}, J=6.9 \mathrm{~Hz}), 4.12-4.16(\mathrm{~m}, 1 \mathrm{H}), 3.20($ broad s, $1 \mathrm{H}), 4.78-4.85(\mathrm{~m}, 2 \mathrm{H}), 12.80($ broad s, $1 \mathrm{H}) .{ }^{13} \mathrm{C}$ $\operatorname{NMR}\left(\mathrm{CDCl}_{3}, 50.3 \mathrm{MHz}\right.$, major isomer): $\delta 178.7,178.7,102.0,76.2,20.7,18.2,17.3 . \mathrm{IR}\left(\mathrm{CHCl}_{3}\right) v$ 3018, 1701, 1651, 1629, 1450, $1348 \mathrm{~cm}^{-1}$. Anal. Calcd for $\mathrm{C}_{8} \mathrm{H}_{10} \mathrm{O}_{3}$ : C, 56.69; H, 5.55. Found: C, 56.70; H, 5.26. MS, $m / z$ (relative intensities) $254\left(\mathrm{M}^{+}, 11\right), 239$ (31), 141 (15), 146 (16), 69 (32), 68 (100), 53 (21).

4-Hydroxy-5-propyl-5H-furan-2-one (6b): $\mathrm{Mp} 74.5-76.5{ }^{\circ} \mathrm{C} .{ }^{1} \mathrm{H} \mathrm{NMR}\left(\mathrm{CDCl}_{3}, 400 \mathrm{MHz}\right): \delta 0.95(\mathrm{t}$, $3 \mathrm{H}, J=7.4 \mathrm{~Hz}), 1.42-1.51(\mathrm{~m}, 2 \mathrm{H}), 1.61-1.68(\mathrm{~m}, 1 \mathrm{H}), 1.91-1.98(\mathrm{~m}, 1 \mathrm{H}), 4.84(\mathrm{dd}, 1 \mathrm{H}, J=7.7,3.7$ $\mathrm{Hz}), 5.06$ (s, 1H). ${ }^{13} \mathrm{C} \mathrm{NMR}\left(\mathrm{CDCl}_{3}, 50.3 \mathrm{MHz}\right): \delta 184.1,178.3,88.7,80.7,33.3,17.5,13.5 . \mathrm{IR}\left(\mathrm{CHCl}_{3}\right)$ v 3030, 2966, 1807, 1759, $1633 \mathrm{~cm}^{-1}$. Anal. Calcd for $\mathrm{C}_{7} \mathrm{H}_{10} \mathrm{O}_{3}: \mathrm{C}, 59.15 ; \mathrm{H}, 7.09$. Found: C, 59.21; $\mathrm{H}$, 7.08. MS, $m / z$ (relative intensities) $142\left(\mathrm{M}^{+}, 4.1\right), 100$ (100), 99 (22), 72 (35), 71 (67), 57 (10). Dilactone 7b: Mixture of diastereomers. Mp 132.5-135.0 ${ }^{\circ} \mathrm{C} .{ }^{1} \mathrm{H}$ NMR $\left(\mathrm{CDCl}_{3}, 400 \mathrm{MHz}\right): \delta 0.88(\mathrm{t}$, $3 \mathrm{H}, J=7.3 \mathrm{~Hz}), 0.96(\mathrm{t}, 6 \mathrm{H}, J=7.3 \mathrm{~Hz}), 1.21-1.26(\mathrm{~m}, 2 \mathrm{H}), 1.36-1.60(\mathrm{~m}, 4 \mathrm{H}), 1.60-1.69(\mathrm{~m}, 2 \mathrm{H}), 1.69-$ 1.75 (m, 2H), 1.91-1.99 (m, 2H), 4.04-4.10 (m, 1H), 2.80 (broad s, 1H), 4.75-4.78 (m, 2H), 12.17 (broad $\mathrm{s}, 1 \mathrm{H}) .{ }^{13} \mathrm{C} \mathrm{NMR}\left(\mathrm{CDCl}_{3}, 50.3 \mathrm{MHz}\right.$, major isomer): $\delta 178.3,178.2,101.4,79.6,33.9,33.4,25.7,20.8$, 
17.7, 13.7, 13.5. IR $\left(\mathrm{CHCl}_{3}\right) \vee 2963,1698,1648,1629,1466,1652 \mathrm{~cm}^{-1}$. Anal. Calcd for $\mathrm{C}_{8} \mathrm{H}_{10} \mathrm{O}_{3}: \mathrm{C}_{\text {, }}$ 63.89; H, 7.74. Found: C, 63.84; H, 7.74. MS, $m / z$ (relative intensities) $338\left(\mathrm{M}^{+}, 1.0\right), 295$ (15), 154 (34), 100 (20), $81(17), 71(17), 68$ (100).

4-Hydroxy-5-isopropyl-5H-furan-2-one (6c): $\mathrm{Mp} 96.5-98.0{ }^{\circ} \mathrm{C} .{ }^{1} \mathrm{H}$ NMR $\left(\mathrm{CDCl}_{3}, 400 \mathrm{MHz}\right): \delta 0.86$ $(\mathrm{d}, 3 \mathrm{H}, J=6.9 \mathrm{~Hz}), 1.08(\mathrm{~d}, 3 \mathrm{H}, J=7.0 \mathrm{~Hz}), 2.17-2.27(\mathrm{~m}, 1 \mathrm{H}), 4.72(\mathrm{~d}, 1 \mathrm{H}, J=3.1 \mathrm{~Hz}), 5.09(\mathrm{~s}, 1 \mathrm{H})$. ${ }^{13} \mathrm{C} \mathrm{NMR}\left(\mathrm{CDCl}_{3}, 50.3 \mathrm{MHz}\right): \delta 183.3,178.5,89.6,84.8,29.5,18.6,14.7 . \mathrm{IR}\left(\mathrm{CHCl}_{3}\right) \vee 3018,2971$, 1803, 1759, 1709, $1623 \mathrm{~cm}^{-1}$. Anal. Calcd. for $\mathrm{C}_{7} \mathrm{H}_{10} \mathrm{O}_{3}$ : C, 59.15; H, 7.09. Found: C, 59.46; H, 7.25. MS, $m / z$ (relative intensities) $142\left(\mathrm{M}^{+}, 20\right), 114(80), 100(100), 72(79), 71(67), 69(30)$.

5-tert-butyl-4-Hydroxy-5H-furan-2-one (6d): $\mathrm{Mp}$ 131.5-133.0 ${ }^{\circ} \mathrm{C} .{ }^{1} \mathrm{H} \mathrm{NMR}\left(\mathrm{CDCl}_{3}, 400 \mathrm{MHz}\right): \delta$ $1.06(\mathrm{~s}, 9 \mathrm{H}), 4.53(\mathrm{~s}, 1 \mathrm{H}), 5.04(\mathrm{~s}, 1 \mathrm{H}) .{ }^{13} \mathrm{C} \mathrm{NMR}\left(\mathrm{CDCl}_{3}, 50.3 \mathrm{MHz}\right): \delta 183.9,178.2,90.4,87.9,25.6$, 25.5. IR $\left(\mathrm{CHCl}_{3}\right) \vee 3018,2971,1803,1759,1709,1623 \mathrm{~cm}^{-1}$. Anal. Calcd for $\mathrm{C}_{8} \mathrm{H}_{12} \mathrm{O}_{3}: \mathrm{C}, 61.52 ; \mathrm{H}$, 7.74. Found: C, 61.37; H, 7.73. MS, $m / z$ (relative intensities) $156\left(\mathrm{M}^{+}, 11\right), 100(100), 99$ (57), 72 (63), $71(80)$.

5-But-3-enyl-4-Hydroxy-5H-furan-2-one (6e): $\mathrm{Mp} 53.5-55.0{ }^{\circ} \mathrm{C} .{ }^{1} \mathrm{H} \mathrm{NMR}\left(\mathrm{CDCl}_{3}, 400 \mathrm{MHz}\right): \delta 1.67-$ $1.88(\mathrm{~m}, 2 \mathrm{H}), 2.12-2.21(\mathrm{~m}, 2 \mathrm{H}), 4.77-4.81(\mathrm{~m}, 1 \mathrm{H}), 5.00(\mathrm{~s}, 1 \mathrm{H}), 4.95-5.06(\mathrm{~m}, 2 \mathrm{H}), 5.65-5.78(\mathrm{~m}, 1 \mathrm{H})$. ${ }^{13} \mathrm{C} \mathrm{NMR}\left(\mathrm{CDCl}_{3}, 50.3 \mathrm{MHz}\right): \delta 184.5,178.5,137.2,116.8,89.7,80.7,31.4,29.2 . \mathrm{IR}\left(\mathrm{CHCl}_{3}\right) \vee 3018$, 2930, 1807, 1760, 1644, $1222 \mathrm{~cm}^{-1}$. Anal. Calcd for $\mathrm{C}_{8} \mathrm{H}_{10} \mathrm{O}_{3}$ : C, 62.33; H, 6.54. Found: C, 62.46; $\mathrm{H}$, 6.61. MS, $m / z$ (relative intensities) $154\left(\mathrm{M}^{+}, 0.8\right), 110(100), 83$ (12), 72 (18), 55 (54).

5-Benzyloxymethyl-4-Hydroxy-5H-furan-2-one (6f): $\mathrm{Mp}$ 65.0-67.0 ${ }^{\circ} \mathrm{C} .{ }^{1} \mathrm{H} \mathrm{NMR}\left(\mathrm{CDCl}_{3}, 400 \mathrm{MHz}\right)$ : $\delta 3.06(\mathrm{~d}, 1 \mathrm{H}, J=22.5 \mathrm{~Hz}), 3.17(\mathrm{~d}, 1 \mathrm{H}, J=22.5 \mathrm{~Hz}), 3.79-3.88(\mathrm{~m}, 2 \mathrm{H}), 4.47(\mathrm{~d}, 1 \mathrm{H}, J=12.2 \mathrm{~Hz}), 4.54$ $(\mathrm{d}, 1 \mathrm{H}, J=12.2 \mathrm{~Hz}), 4.74-4.76(\mathrm{~m}, 1 \mathrm{H}), 7.26-7.35(\mathrm{~m}, 5 \mathrm{H}) .{ }^{13} \mathrm{C} \mathrm{NMR}\left(\mathrm{CDCl}_{3}, 50.3 \mathrm{MHz}\right): \delta 204.2$, $170.3,136.7,128.5,128.0,127.5,85.3,73.7,68.4,38.2 . \mathrm{IR}\left(\mathrm{CHCl}_{3}\right) \vee 3018,2930,2866,1807,1760$, 1672, $1225 \mathrm{~cm}^{-1}$. Anal. Calcd for $\mathrm{C}_{12} \mathrm{H}_{12} \mathrm{O}_{4}: \mathrm{C}, 65.45 ; \mathrm{H}, 5.49$. Found: C, 65.69; H, 5.42. MS, $\mathrm{m} / z$ (relative intensities) $220\left(\mathrm{M}^{+}, 12\right), 107(13), 91(100), 65(7.8)$. 
Acknowledgment. This research was supported by the Spanish Ministerio de Ciencia y Tecnología

(PB98-0443-C02-02 and PPQ2002-04361-C04-03). DTA thanks the Instituto Canario de Investigación

del Cáncer for financial support.

\section{References and Footnotes.}

\footnotetext{
${ }^{\dagger}$ Instituto de Productos Naturales y Agrobiología

\# Instituto Canario de Investigación del Cáncer

^ On leave from Universidad de La República, Uruguay

${ }^{ \pm}$Associate researcher at the Instituto Canario de Investigación del Cáncer

* Instituto Universitario de Bioorgánica Antonio González

${ }^{\S}$ On leave from Universidad Autónoma de Santo Domingo, República Dominicana

${ }^{1}$ Haynes, L.J.; Plimmer, J. R. Q. Rev. Chem. Soc. 1960, 14, 292-315.
}

${ }^{2}$ (a) Sodeoka,M.; Sampe, R.; Kojima, S.;Baba,Y.;Usui, T.; Ueda, K.; Osada, H.; J. Med. Chem. 2001, 44, 3216-3222; (b) Bühler, H.; Bayer, A.; Effenberg, F. Chem. Eur. J. 2000, 6, 2564-2571 and references cited therein.

${ }^{3}$ (a) Mitsos, C. A.; Zografos, A. L.; Igglessi-Markopoulou, O. J. Org. Chem. 2000, 65, 5852-5853; (b) Ge, P.; Kirk, K. L. J. Org. Chem. 1996, 61, 8671-8673; (c) Hori, K.; Kazuno, H.; Nomura, K.;Yoshii, E. Tetrahedron Lett. 1993, 24, 2183-2186; (d) Witiak, D. T.; Tehim, A. K. J. Org. Chem. 1990, 55, 1112-1114; (e) Booth, P. M.; Fox, C. M. J.; Ley, S. V. J. Chem. Soc., Perkin Trans. 1 1987, 121-129; (f) Brandänge, S.; Flodman, L.; Norberg, A. J. Org. Chem. 1984, 49, 927-928; (g) Ireland, R. E.; Thompson, W. J. J. Org. Chem. 1979, 44, 3041-3052; (h) Bloomer, J. L.; Kappler, F. E. J. Chem. Soc.,Perkin Trans. 1 1976, 1485-1491; (i) Bell, P. M.; Sørensen, E.; Balieu, E. Acta Chem. Scand. 1968, 22, 3251-3255.

${ }^{4}$ (a) Takabe,K.; Mase, N.; Nomoto, M.; Daicho, M.; Tauchi, T.; Yoda, H. J. Chem. Soc., Perkin Trans. 1 2002, 500-502; (b) Momose, T.; Toyooka, N.; Takeuchi,Y. Heterocycles 1986, 24, 1429-1431; (c) Svendsen, A.; Boll, P. M. Tetrahedron 1973, $29,4251-4258$.

${ }^{5}$ Synthesized using the Claisen condensation: (a) Brandaenge, S.; Flodman, L.; Norbreg, A. J. Org. Chem. 1984, 49, 927-928; (b) Pollet, P.; Gelin, S. Tetrahedron 1978, 34, 1453-1455; (c) Damon, R. E.; Luo, T.; Schlessinger, R. H. Tetrahedron Lett. 1976, 32, 2749-2752.

${ }^{6}$ Synthesized using the Blaise reaction with cyanhydrins: (a) Ref. 2b; (b) Duffield,m J. J.;Regan, A. C. Tetrahedron: Asymmetry 1996, 7, 663-666; (c) Veronese, A.; Callegari, R.; Bertazzo, A. Heterocycles 1991, 32, 2205-2215; (d) Kitazume, T. J. Fluorine Chem. 1987, 35, 287-294; (e)Krepski, L. R.;Lynch, L. E.; Heilmann, S. M.; Rasmussen, J. K. Tetrahedron Lett. 1985, 26, 981-984.

${ }^{7}$ (a) Mitra, A.; Yamashita, M.; Kawasaki, I.; Murai, H.; Yoshioka,T.; Ohta, S. Synlett 1997, 8, 909-910; (b) Wrobel, J. E.; Ganem, B. J. Org. Chem. 1983, 48, 3761-3764.

${ }^{8}$ (a) Lieb, F.; Benet-Buchholtz, J.; Facke, T.; Fischer, R.; Graff, A., Lefebvre, I. M.; Stetter, J. Tetrahedron 2001, 57, 41334137; (b) Schobert, R.; Mueller, S.; Bestmann, H.-J. Synlett 1995, 5, 425-426; (c) Sato, M.; Sasaki, J.; Sugita, Y.; Yasuda, S.; Sakoda, H.; Kaneko, C. Tetrahedron 1991, 47, 5689-5706.

${ }^{9}$ Ramage, R.; Griffiths, G. J.; Shutt, F. E.; Sweeney, J. N. A. J. Chem. Soc., Perkin Trans. 1 1984, 1539-1545.

${ }^{10}$ Matso, K.; Sakaguchi, Y. Heterocycles 1996, 43, 2553-2556.

${ }^{11}$ Desmaele, D. Tetrahedron 1992, 48, 2925-2934.

12 Although the method described by Schobert et al. ${ }^{8 b}$ is a true one-pot synthesis of tetronic acid derivatives, the ketenylidenetriphenyl-phosphorane reagent needs to be elaborated. 
${ }^{13}$ de Armas, P.; García-Tellado, F.; Marrero-Tellado, J.J.; Tejedor, D.; Maestro, M. A.; González-Platas, J. Org. Lett. 2001, 3, 1905-1908.

${ }^{14}$ (a) Tietze, L. F.; Haunert, F., in Stimulating Concepts in Chemistry; Shibasaki, M.; Stoddart, J. F.; Vögtle, F., Eds.; WileyVCH: Weinheim, 2000, pp 39-64. (b) Filippini, M. H.; Rodriguez, J. Chem. Rev. 1999, 99, 27-76. (c) Tietze, L. F. Chem. Rev. 1996, 96, 115-136. (d) Bunce, R. A. Tetrahedron 1995, 51, 13103-13160.

${ }^{15}$ (a) Wess, G.; Urmann, M.; Sickenberger, B. Angew. Chem., Int. Ed. 2001, 40, 3341-3350. (b) Arya, P.; Chou, D. T. H.; Baek, M. G. Angew. Chem., Int. Ed. 2001, 40, 339-346. (c) Drews, J. Science 2000, 287, 1960-1964. (d) Schriber, S. L. Science 2000, 287, 1964-1969. (e) Nicolaou, K. C.; Montagnon, T.; Ulven, T.; Baran, P. S.; Zhong, Y.-L.; Sarabia, F. Journal of the American Chemical Society 2002, 124, 5718-5728.

${ }^{16}$ Kresge, A. J.; Pruszynski, P. J. Org. Chem. 1991, 56, 4808-4811.

17 Zimmer, H.; Hillstrom, W. W.; Schmidt, J. C.; Seemuth, P. D.; Vögeli, R. J. Org. Chem. 1978, 43 1541-1544 and references cited therein..

${ }^{18}$ Brohm, D.; Metzger, S.; Bhargava, A.; Müller, O.; Lieb, F.; Waldmann, H. Angew. Chem. Int. Ed. 2002, 41, $307-311$.

19 (a) Hamaguchi, T.; Suda, T.; Osada, H. FEBS Lett. 1995, 372, 54-58. (b) Roggo, B. E.; Hug, P.; Moss, S.; Raschdorf, F.; Peter, H. H.; Roesel, J. J. Antibiotics 1994, 47, 143. (c) Roggo, B. E.; Petersen, F.; Delmendo, R.; Jenny, H.-B.; Peter, H. H.; Roesel, J. J. Antibiotics 1994, 47, 136. 
TOC

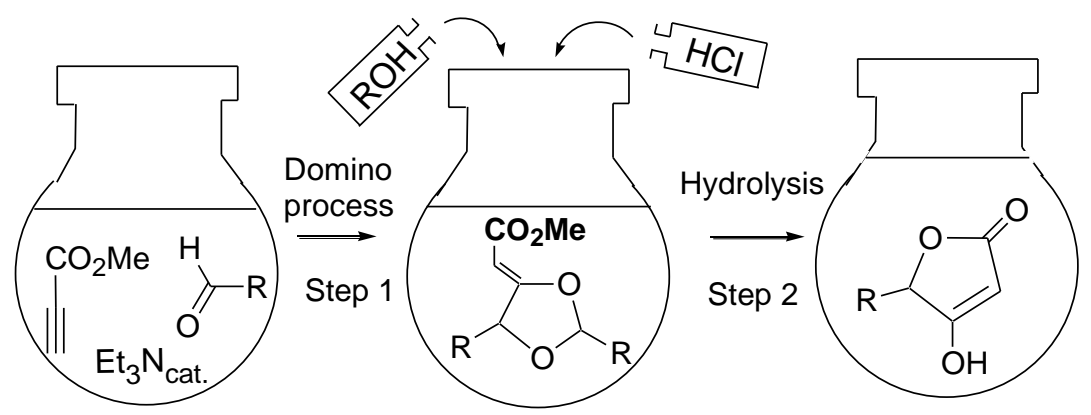

